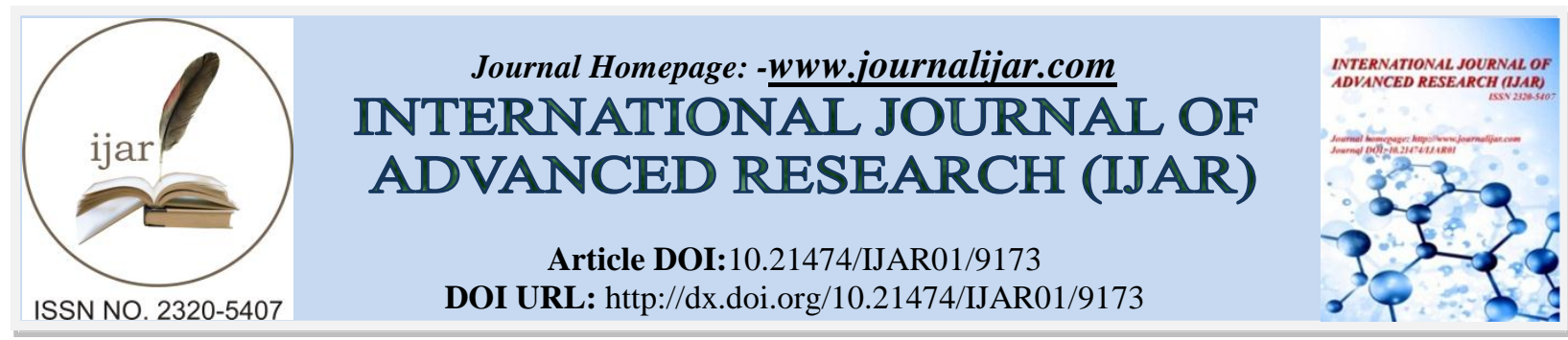

RESEARCH ARTICLE

\title{
INTERDEPENDENCE BETWEEN CHEMICAL SCIENCES AND MEDICAL SCIENCES- AN OVERVIEW.
}

Dr. Subhas Chandra Bhat ${ }^{1}$ and Dr. Ratanadeep Abbas ${ }^{2}$.

1. Assistant professor in chemistry (wbes), govt. College of education, banipur, north 24 parganas.

2. Md anaesthesia and critical care, national medical college and hospital, kolkata, w.b.

\section{Manuscript Info}

Manuscript History

Received: 24 March 2019

Final Accepted: 26 April 2019

Published: May 2019

\section{Abstract:}

Development of Medical Scieneces is mostly depending on developments Chemical Sciences. How far their dependence is presently a matter of study .Doctors should always be careful about the drug compositios and their functions as well as malfunctioning possibilities on human physiology. This article is a study of such an overview for chemists, doctors and common people.

Copy Right, IJAR, 2019,. All rights reserved.

\section{Introduction:-}

It is well known to all that a Medical Practitioner has to deal with all Bio-chemical and Physical aspects of human health. Human body is a mechanism combining of laws and principles of chemistry-physics-mathematics-biology sciences and psychological sciences. A doctor or a medical practitioner has to maintain quite a good knowledge of structure, composition and processes underlying a human body so that any change or deviation of normal physiological condition of human body could be identified properly and easily by him. He can then take needful action using any medicines that are made up of desired chemical composition. Here the doctor has to keep a quite strong knowledge of chemistry or chemical sciences for matching chemistry (chemical composition) of the medicine and chemistry of the body (of the patient) under changed condition. In this article, central point of discussion is to how far the knowledge of chemical sciences is useful to medical sciences and specially to a medical practitioner as well as to a common people who are attached to these sciences by any way in their day to day life.

\section{Discussion:-}

It is observed from the curriculum of medical entrance examination that a student must have detailed knowledge of chemical science and biological science and their inter-relationship. Chemistry subject itself has different branches viz. physical, inorganic and organic, analytical and industrial etc. Organic chemistry has more important role in designing therapeutic drugs with the development and discovery of new medicinal aspect of natural and synthetic ones. Generally, drugs are chemical substances having physiological effects and are also used as medicine prescribed by medical practitioner for remedy of different diseases. Drugs have some specific toxic effects on 'microbes' those are causes for disease and may destroy the neo-plastic tissues by acting as chemo-therapeutic agent on it.Here lies the role of medical practitioner to choose justified drug or medicine for properly removing the effects of germs. Both tramadol and heroin are medicine of painkiller but, regular consumption of heroin as a painkiller may cause addiction and intoxication whereas tramadol uses do not have such effect. Here lies the role of knowledge of both these sciences for judicious uses of medicine on human body. 


\section{Some more examples related to these:}

(1) Due to over eating or mal-digestion extra acidity is formed within our body. But knowledge of acid base neutralization concept of chemistry helps a doctor to prescribe any antacid of mild dose (of alkali) to neutralize the excess hydrochloric acid secreted in stomach.

(2) If any one consumes oily and fatty food regularly, there is a possibility of forming cholesterol (Molecular Formula- $\mathrm{C}_{27} \mathrm{H}_{46} \mathrm{O}$ having molecular mass is of 386.664 units) in blood vessel called Hypercholesterolemia, which hinders the normal blood flow from heart to all over the human body and possibility of heart attack increases thereby. In this problem the doctor suggests some medicine to melt or dissolve the cholesterol and maintain the blood fluidity. This in turn prevents risk of coronary artery disease and thromboembolic episode.

(3) Generally sodium and potassium are very much fast moving ions which maintain electrolytic balance and membrane electro-neutrality in our body. Besides this sodium ions plays the major role as extra-cellular cations whereas potassium ions plays the role as intra-cellular cations. Sodium and potassium ions level in cells also maintain various physiological phenomenon like cardial contraction $\left(\mathrm{K}^{+}\right)$, serum osmolarity $\left(\mathrm{Na}^{+}\right)$. So deficit of sodium and potassium may result in loss of memory of a person. At this time, doctors use the knowledge of chemistry of fast moving ions and suggest the medicines (Sodium or potassium salt of weak organic acid) to increase the sodium and potassium ions level of blood.

(4) In case of dehydration of a patient, doctors prescribe oral rehydration by using either homemade saline water prepared with common salt and sugar solution time to time or inject saline water by channel to maintain the sufficient sodium and carbohydrate with water level in the blood so that organs of patient can function at its minimum or normal level.

(5) In case of bacterial or fungal infection of a body some metabolic or biochemical changes occur to a localized area or whole body. In this case, normal physical immunity of a body fails to recover to its normal function. According to medical science doctors are compelled to use antibiotic or anti fungal medicine to remove the germs and recover the normal function of the organ.

(6)Sometimes, oral consumption of any kind of poison by any person causes a serious challenge to a doctor both physically and mentally. In this case, Chelating (Means Caging the metal ions or poisonous ions by cyclic polydentate radical or agent) knowledge of complex chemistry is very much essential so that s/he can suggest the proper drug or complex ligand (metal binding agent) that binds with the poison to decrease the toxic effect of it.

(7) Presently in case of treatment of Cancer and AIDS it is observed that a serious research is going on all over the world to find out a chemical drug or medicine that can prevent the malignant character of the carcinogenic cells by chemotherapy and increase the immunity power of the body to prevent AIDS.

\section{More Inter-relationships:}

Day to day uses of therapeutic action of different drugs and medicines prescribed by medical practitioners are classified as : i) Common Analgesics, ii) Anesthesia, iii) Tranquillizers, iv) Common antiseptic and disinfectant, v) Antimicrobials, vi) Antifertillity drug, vii) Antibiotics, viii) Antacid, ix) Antihistamines, and x) Antioxidants. All the organic-chemical drugs are of either synthetically prepared or naturally extracted from plants. Inorganic compounds like boric acid, potassium permanganate, sodium bicarbonate, iodine solution etc. are industrially prepared from the different inorganic sources. A brief discussion will help one to understand the role of chemical sciences to a medical practitioner for giving the better treatment. To avoid complexity in writing chemical formula of the compound only common name are used here.

i) Common Analgesics: Analgesics are such chemical substances that temporarily reduce the pain of patients. Those analgesics are also used in decreasing the fever or temperature of human body called antipyretic analgesic like paracetamol, fencitine, analgine, aspirin etc. Other kinds of analgesics are narcotics by which sleep or unconsciousness is produced among the patients. Therefore these are restricted drug like heroin, methadone, codeine etc.Generally analgesics are sometime used as painkiller. As for example Ibuprofen, Phenyl butazone are used as painkiller but they have no power to relieve from fever.

ii) Anesthesia: In case of surgery to minimize the feeling of pain anesthetics are used. They are of two type viz. General and Local. General anesthetics are ketamine propofol etomidate, or sodium pentathal. Local anesthetics act on voltage grated $\mathrm{Na}^{+}$and decreases Action Potencial(AP) propagation. These are xylocane 
gel, ethyl chloride as spray \& procane as injection. In earlier days Chloroform and ether compounds were used as anesthetics but due to developments in chemical science and medical science these are not used in present days.

iii) Tranquillizers: Tranquillizers are psychotherapeutic drug which deactivate the central nervous system, mental pressure and emotional exaggeration of patients. To avoid despair due to lack of Nor-adrenaline neurotransmitter hormone of patients medical practitioners generally prescribed Iproniazid or phenelzine. Barbituric acid, luminal, veronal and seconal mostly used as tranquillizers. All these are specially designed research product of chemistry.

iv) Common Antiseptic and Disinfectant: Antiseptics are generally used to counter sepsis whereas disinfectants are used to destroy the microorganism on inanimate substances. The antiseptic creams are furacine, soframycine etc. In Medical Science, disinfectants are of two categories, one is for surface sterilization and second one is for inanimate objects. Alcohol or spirit, iodine, mercurochrome, aldehydes, acids, cresol, dettol, sallol, savlon, boric acid, are used as surface sterilization whereas, bleaching powder, phenol, chlorine, iodoform, potassium permanganate, all these are used as disinfectant for inanimate objects..

Similarly, for other kind of diseases many steroids are used as antimicrobials, antifertility drug. Many antibiotics, antacids like milk of magnesia, antihistamine like seldane, anti oxidant like vitamin ' $E$ ' present in citric acid, ascorbic acids etc. are used by medical practitioners which are products of chemical laboratory. So, it may be said that sound knowledge of chemistry is very much essential to the medical practitioners, by which he or she can help, guide and treat the patients properly. Thus biochemical knowledge of human body and common laws and principles of chemistry helps a doctor a lot in his or her profession.

The chemicals used in preparing a medicine are generally displayed on its cover. The same medicine with same chemical compositions may be produced by different companies but with different brand names. A patient might recover when a doctor prescribes a proper medicine with a certain chemical compositions under any brand name. However it is the chemical composition of medicine that matters, brand name of medicine is secondary altogether.

\section{Acknowledge:-}

The authors are thankfully acknowledges the cooperation and guidance received from Dr. Kamal Krishna De, President of AISTA,WB, Mrs. Bakul Bhat and Mrs.Tania Abbas while writing this article.

\section{References:-}

1. Text Book of Biochemistry (Second edition, 2013), Kondreaddy Rambabu et.al. AITBS Publishers, India.

2. The fundamental principles of organic chemistry (Vol.I\&II), I. L. Finar (2016) ELBS, India.

3. Uchhatara Rasayan' (Vol.-II), A Bengali Text Book Higher Secondary Chemistry, Bhattacharya et, al., Santra Publication, (2018), Kolkata.

4. Higher Secondary Chemistry (Vol.-II), Maity et. al., Chhaya Prakasani,(2018) Kolkata.

5. Jibavidya' A Bengali Text Book of Biology of Higher Secondary Level, Bardhan et.al. Calcutta Book House,( 2018), Kolkata.

6. WWW. Wikipedia and WWW. Google (Date of retrieval 01.05.2019).

7. VIGYAN SIKSHAK(Vol-3,June,2019), Annual journal of AISTA,Kolkata-25, W.B. 